\title{
Sensitivity in Multi-Ensemble Scheduling
}

\author{
Jörg Bremer \\ Department of Computing Science \\ Carl von Ossietzky University \\ Oldenburg, Germany \\ joerg.bremer@uni-oldenburg.de
}

\author{
Sebastian Lehnhoff \\ Department of Computing Science \\ Carl von Ossietzky University \\ Oldenburg, Germany \\ sebastian.lehnhoff@uni-oldenburg.de
}

\begin{abstract}
Future smart grid control demands delegation of liabilities to distributed, rather small energy resources in contrast to today's traditional large control power units. Distributed energy scheduling constitutes a complex task for optimization algorithms regarding the underlying high-dimensional, multimodal and nonlinear problem structure. For predictive scheduling with high penetration of renewable energy resources, agent-based approaches using classifier-based decoders for modeling individua flexibilities have shown good performance. On the other hand, such decoder-based methods are currently designed for single entities and not able to cope with ensembles of energy resources. Aggregating training sets sampled from individually modeled energy units results in folded distributions with unfavorable properties for training a decoder. Nevertheless, this happens to be a quite frequent use case, e.g. when a hotel, a small business, a school or similar with an ensemble of co-generation, heat pump, solar power, and controllable consumers wants to take part in decentralized predictive scheduling. Recently, an extension to an established agent approach for scheduling individual single energy units has been proposed that is based on second level optimization. The agents' decision routine may be enhanced by a covariance matrix adaption evolution strategy that is hybridized with decoders. In this way, locally managed ensembles of energy units can be included. The applicability has already been demonstrated, but the effects of ensemble composition are so far unknown. Here, we give an widened view on the underlying power level distribution problem and extend the results by conducting a sensitivity analysis on the impact of ensemble size and penetration on communication overhead and residual error.
\end{abstract}

\section{INTRODUCTION}

In Germany where a financial security of guaranteed feed-in prices has meanwhile been granted since the early 90th - but also in other countries of the European union and world wide, the share of distributed energy resources (DER) within the electricity grid is constantly and rapidly rising. According to the goal defined by the European Commission [1], concepts for integration into electricity markets will quickly become indispensable to reduce subsidy dependence for both: active power provision and for providing ancillary services like frequency or voltage control [2], [3].

Consequently, combining smart measurement technologies for decentralized information gathering on current operational grid state, new tele-control techniques, communication standards and scalable, decentralized self-organized control schemes will lead to a so called smart grid with decentralized power conditioning and control of the production and distribution of electricity managed without central control; as in the vision of [4] or similar for Europe [5].

As the smart grid will have to delegate many control tasks to small and distributed energy units, new control algorithms are required that are able to cope with large problem sizes and distributed and only locally available information. Virtual power plants (VPP) are a well-known instrument for aggregating and controlling DER [6]. Concepts for several purposes (commercial as well as technical) have been developed. A usual use case commonly emerging within VPP control is the need for scheduling the operation of participating DER. Predictive scheduling [7] describes the optimization problem for day-ahead planning of energy generation in VPPs, where the goal is to select a schedule for each energy unit - from an individual search space of feasible schedules with respect to a future planning horizon - such that a global objective function (e.g. resembling a target power profile for the VPP as close as possible) is optimized.

Recently, distributed approaches gained more and more importance for VPP control. Different works proposed hierarchical and decentralized architectures based on multi-agent systems and market-based computing [8], [9]. Newer approaches try to establish self-organization between actors within the grid [10]-[12]. In contrast, today's commercial VPP are often operated by a single authority that at the same time is the owner of (and responsible for) all distributed energy resources in this rather static unit ensemble. Independently from a concrete implementation for predictive scheduling, the dispatch algorithm has to choose a schedule for each DER in the VPP such that all objectives are met.

In order to choose an appropriate schedule for each participating DER, the algorithm must know from each DER, which schedules are actually operable and which are not. Depending on the type of DER, different constraints restrict possible operations. The information about individual local feasibility of schedules has to be modeled appropriately in (distributed) optimization scenarios, in order to allow unit independent algorithm development. For this purpose, metamodels of constrained spaces of operable schedules have been shown indispensable as a means for independently modeling constraints and feasible regions of flexibility. Each energy unit has its own individual flexibility - i.e. the set of schedules that might be operated without violating any technical operational constraint - based on the capabilities of the unit, 


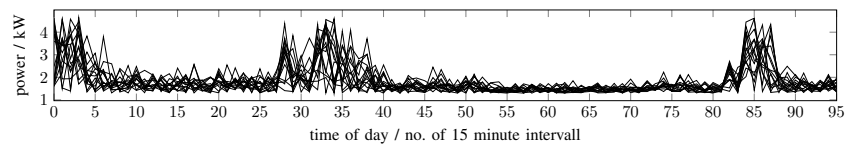

Fig. 1. Example for a training set of schedules for a co-generation plant. A state-of-charge of $50 \%$ at night and an increased thermal demand for showering in the morning and dish washing in the evening result in higher flexibilities during these periods.

operation conditions (weather, etc.), cost restrictions and so forth. Integrating these constraints to possible operations of an arbitrary energy unit demands a means for meta-modeling that allows model independent access to feasibility information. [13] introduced a support vector based model that captures individual feasible regions from training sets of operable example schedules. Figures 1 and 2 show example training sets for a co-generation plant and a heat pump respectively.

With an appropriate extension - so called decoders [14] - , these models can also be used for repairing infeasible solution or for systematically generating feasible solutions [15]. Agentbased approaches can derive a so called support vector decoder automatically from the surrogate model and use it as a means for generating feasible solutions without domain knowledge on the (possible, situational) operations of the controlled energy resource [14].

Examples for using decoders in optimization within the smart grid can be found in [16]-[19]. In general, the idea works in two successive stages - a decoder training phase and the actual algorithm/ negotiation execution phase where the decoder is used [7]. During the training phase a decoder is calculated for each unit. These calculations can be done fully parallel. During the succeeding load planning phase, these decoders may be used by an optimization algorithm that determines the optimal partition of a given active power target schedule into schedules for each single unit. The decoder automatically repairs infeasible solutions and thus the solver does not need any domain knowledge about the energy units, their individual constraints, or possible operation.

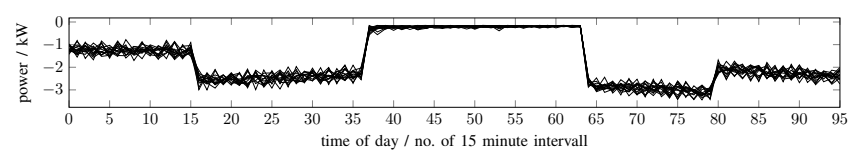

Fig. 2. Example for a training set of schedules for a heat pump with a maximum deviation of $500 \mathrm{Wh}$ from the integral of set thermal demand.

An example for a recently developed agent approach for fully decentralized predictive scheduling is given by the combinatorial heuristics for distributed agents (COHDA). In COHDA [20] each agent is responsible for exactly one energy unit and uses a decoder to locally decide on feasible schedules for the represented unit. The algorithm has shown excellent performance [17], [20], [21]. But, as soon as an agent has to represent a local ensemble of energy units instead of a single device, a problem arises because usually only flexibility models of single units are available. Generating a single decoder for handling all constraints and feasible operations of the whole ensemble is hardly possible due to statistical problems when combining training sets from individually sampled flexibility models. Due to the folded densities only a very small portion from the interior of the feasible region (the dense region) is captured by the machine learning process. But, a combined training set is needed if one wants to train a single decoder for each agent.

For this reason, in [22] a substitute for the single decoder part that generates suitable and feasible schedules for the negotiating agents has been proposed. To achieve this, an evolution strategy is harnessed to do the job of solving the problem using individual decoders (one for each unit in the ensemble). In this way, an optimization problem has to be solved instead of a single mapping with a decoder for each agent decision during the negotiation, but with harnessing the full flexibility of the ensemble. Hence, a new decision method is introduced to the agent approach based on a covariance matrix adaption evolution strategy that widens the applicability to including multiple local ensembles of DER into the VPP without changing the underlying negotiation between the agents.

In [22] the general approach had been scrutinized on basis of gained optimization results regarding effect and performance of the CMA-ES part. A closer look on underlying mechanisms of the agent negotiation and parameter impact is missing so far. Moreover, the influence of ensemble size and composition is unknown. Here, we extend the former work with a study on the impact of folded distributions on different energy units' aggregated flexibility - constituted by the agents' entities - and conduct a sensitivity analysis regarding the impact of group size, composition or group number on the agent approach as well as on the CMA-ES intermediate results.

The rest of the paper is organized as follows. First, an outline on predictive scheduling and related work regarding the decoder approach as well as decentralized, agent-based methods for solving is presented. A strong focus is on the combinatorial heuristics for distributed agents. After scrutinizing the problem of folded power level distributions in aggregated training sets for different types of energy unit ensembles, the necessity of integrating a heuristic approach into the agent method for ensembles is derived. We recap the hybridization of covariance matrix adaption evolutions strategies with support vector decoders and the bi-level approach from [22] for circumventing the problem of folded distributions. The sensitivity of different group traits is analyzed. We conclude with results from several simulation studies showing beyond the effectiveness of the hybrid approach the scalability regarding ensemble size, penetration and communication expenses. Moreover, it is shown that the overall efficiency of the underlying agent approach is not seriously effected by integrating a suboptimization process into the decision phase. 


\section{RELATED WORK}

\section{A. Predictive scheduling}

As related work, solutions to predictive scheduling with decoders have to be discussed in the context of agent-based approaches prior to deriving the root cause that raises the problem when extending scheduling to participants that locally have to control more than one single energy unit. We start with a definition of the general predictive scheduling problem.

As opposed to the usual time series model, we regard a schedule as real valued vector $\boldsymbol{p}=\left(p_{1}, \ldots, p_{d}\right) \in \mathbb{R}^{d}$ with each element $p_{j}$ denoting mean active power generated (positive values) or consumed (negative values) during the $j$ th of $d$ time intervals. Starting time and width of each time interval are assumed to be known from context information. The feasibility of a schedule $\boldsymbol{p}$ is defined by sets of unit specific technical and economic constraints.

One of the crucial challenges in operating a VPP arises from the complexity of the scheduling task due to the large amount of (small) energy units in the distribution grid [23]. In the following, we consider predictive scheduling, where the goal is to select exactly one schedule $\boldsymbol{p}_{i}$ for each energy unit $U_{i}$ from a search space $\mathcal{F}^{(U)}$ of feasible schedules specific to the possible operations and technical constraints of unit $U$ and with respect to a future planning horizon, such that a global objective function (e.g. resembling a target power profile) is optimized by the sum of individual contributions [24]. A basic formulation of the scheduling problem is given by

$$
\delta\left(\sum_{i=1}^{m} \boldsymbol{p}_{i}, \boldsymbol{\zeta}\right) \rightarrow \min ; \text { s.t. } \quad \boldsymbol{p}_{i} \in \mathcal{F}^{\left(U_{i}\right)} \forall U_{i} \in \mathcal{U} .
$$

In equation (1) $\delta$ denotes an (in general) arbitrary distance measure for evaluating the difference between the aggregated schedule of the group and the desired target schedule $\zeta$. W. l.o.g. we assume the Euclidean distance is used.

To each energy unit $U_{i}$ exactly one schedule $\boldsymbol{p}_{i}$ has to be assigned. The desired target schedule is given by $\zeta$. $\mathcal{F}^{\left(U_{i}\right)}$ denotes the individual set of feasible schedules that are operable for unit $U_{i}$ without violating any (technical) constraint. Solving this problem without unit independent constraint handling leads to specific implementations that are not suitable for handling changes in VPP composition or unit setup without having changes in the implementation of the scheduling algorithm [17].

Flexibility modelling can be understood as the task of modelling constraints for energy units. For optimization approaches in smart grid scenarios, black-box models capable of abstracting from the intrinsic model have proved useful [25], [26]. They do not need to be known at compile time. A powerful, yet flexible way of constraint-handling is the use of a decoder that gives a search algorithm hints on where in the search space to look for schedules satisfying local hard constraints (feasible schedules) [26], [27].

For our experiments, we used a decoder as described in [15]. Here, a decoder $\gamma$ is given as mapping function

$$
\gamma: \mathbb{R}^{d} \rightarrow \mathbb{R}^{d} ; \gamma(\boldsymbol{p}) \mapsto \boldsymbol{p}^{*}
$$

With $\boldsymbol{p}^{*}$ having the following properties:

- $\boldsymbol{p}^{*}$ can be operated by the respective energy unit without violating any constraint,

- the distance $\left\|\boldsymbol{p}-\boldsymbol{p}^{*}\right\|$ is small; where the term small depends on the problem at hand and often denotes the smallest distance of $\boldsymbol{p}$ to the feasible region.

With such decoder concept for constraint handling one can now reformulate the optimization problem as

$$
\delta\left(\sum_{i=1}^{m} \gamma_{i}\left(\boldsymbol{p}_{i}\right), \boldsymbol{\zeta}\right) \rightarrow \min ,
$$

where $\gamma_{i}$ is the decoder function of unit $i$ that produces feasible, schedules from $\boldsymbol{p} \in\left[0, p_{\max }\right]^{d}$ resulting in schedules that are operable by that unit. Please note, that this is a constraint free formulation. With this problem formulation, many standard algorithms for optimization can be easily adapted as there are no constraints (apart from a simple box constraint $\boldsymbol{p} \in\left[0, p_{\max }\right]^{d}$ ) to be handled and no domain specific implementation (regarding the energy units and their operation schedules) has to be integrated. Equation (3) is used as a surrogate objective to find the solution to the constrained optimization problem equation (1).

\section{B. COHDA}

The Combinatorial Optimization Heuristics for Distributed Agents (COHDA) was originally introduced in [28], [29]. Since then it has been applied to a variety of smart grid applications [17], [24], [30], [31]. With our explanations we follow [29].

Originally, COHDA has been designed as a fully distributed solution to the predictive scheduling problem (as distributed constraint optimization formulation) in smart grid management [28]. In this scenario, each agent in the multi-agent system is in charge of controlling exactly one distributed energy resource (generator or controllable consumer) with procuration for negotiating the energy. All energy resources are drawn together to a virtual power plant and the controlling agents form a coalition that has to control the VPP in a distributed way. It is the goal for the predictive scheduling problem to find exactly one schedule for each energy unit such that

1) each assigned schedule can be operated by the respective energy unit without violating any hard technical constraint, and

2) the difference between the sum of all targets and a desired given target schedule is minimized.

The target schedule usually comprises 96 time intervals of 15 minutes each with a given amount of energy (or equivalently mean active power) for each time interval, but might also be constituted for a shorter time frame by a given energy product that the coalition has to deliver.

An agent in COHDA does not represent a complete solution as it is the case for instance in population-based approaches [32], [33]. Each agent represents a class within a multiple choice knapsack combinatorial problem [34]. Applied to predictive scheduling each class refers to the feasible region in 
the solution space of the respective energy unit. Each agent chooses schedules as solution candidate only from the set of feasible schedules that belongs to the DER controlled by this agent. Each agent is connected with a rather small subset of other agents from the multi-agent system and may only communicate with agents from this limited neighborhood. The neighborhood (communication network) is defined by a small world graph [35]. As long as this graph is at least simply connected, each agent collects information from the direct neighborhood and as each received message also contains (not necessarily up-to-date) information from the transitive neighborhood, each agent may accumulate information about the choices of other agents and thus gains his own local belief of the aggregated schedule that the other agents are going to operate. With this belief, each agent may choose a schedule for the own controlled energy unit in a way that the coalition is put forward best while at the same time own constraints are obeyed and own interests are pursued.

All choices for own schedules are rooted in incomplete knowledge and beliefs in what other agents are probably going to do; gathered from received messages. The taken own choice (together with the basis for decision-making) is communicated to all neighbors and in this way knowledge is successively spread throughout the coalition without any central memory. This process is repeated. Because all spread information about schedule choices is labeled with an age, each agent may decide easily whether the own knowledge repository has to be updated. Any update results in recalculating of the own best schedule contribution and spreading it to the direct neighbors. By and by all agents accumulate complete information and as soon as no agent is capable of offering a schedule that results in a better solution, the algorithm converges and terminates. Convergence has been proven in [20].

More formally, each time an agent receives a message, three successive steps are conducted. First, during the perceive phase an agent $a_{j}$ updates its own working memory $\kappa_{j}$ with the received working memory $\kappa_{i}$ from agent $a_{i}$. From the foreign working memory the objective of the optimization (i.e. the target schedule) is imported (if not already known) as well as the configuration that constitutes the calculation base of a neighboring agent $a_{i}$. An update is conducted if the received configuration is larger or has achieved a better objective value. In this way, schedules that reflect the so far best choices of other agents and that are not already known in the own working memory are imported from the received memory.

During the following decision phase agent $a_{j}$ has to decide on the best choice for his own schedule based on the updated belief about the system state $\Gamma_{k}$. Index $k$ indicates the age of the system state information. The agent knows which schedules of a subset of other agents (or all) are going to operate. Thus, the schedule that fills the gap to the desired target schedule exactly can be easily identified. Due to operational constraints of the controlled DER, this optimal schedule can usually not be operated. Thus, each agent is equipped with a decoder that automatically maps the identified optimal schedule to a nearby feasible schedule that is operable by the
DER and thus feasible. In this way, the decision routine of the agent reduces simply to a mapping call of the decoder. Based on a set of feasible schedules sampled from an appropriate simulation model for flexibility prediction [36], the decoder can be built by learning a support vector model after the approach of [15].

If the objective value for the configuration with this new candidate is better, this new solution candidate is kept as selected one. Finally, if a new solution candidate has been found, the working memory with this new configuration is sent to all agents in the local neighborhood. The procedure terminates, as soon as all agents reach the same system state and no new messages are generated. In this case no agent is able to find a better solution. Finally, all agents know the same final result.

As the whole procedure is based exclusively on local decisions, each agent decides privately which schedules are taken. Private interest and preferences can be included and all information on the flexibility of the local DER is kept private. The same must hold true for agents controlling an ensemble of energy units.

\section{ENSEMBLE SCHEDULING}

\section{A. Problem}

Sometimes the technical equipment of a single participant in a virtual power plant consists of more than just a single generator (or prosumer or controllable consumption). Nevertheless, the owner as operator is usually still represented by a single controlling agent when embedded into a decentralized agent-based control scheme inside a virtual power plant. In this case that agent has to handle the ensemble of energy units as a single unit (in a sense as a single sub VPP) and negotiate to the other agents with the aggregated flexibility. Nevertheless, there is usually no joint model of the whole ensemble, and thus the agent has to use an individual model of each unit and thus a set of individual decoders for deciding on an aggregated schedule for the ensemble.

If an agent covers a set of energy units instead of a single unit, a decoder for the joint feasible region of the group of units has to be used. A model of the operation of the ensemble of units is usually not available. Using the training sets of individual energy units and randomly combining them (adding up exactly one from each training set) to joint schedules in order to gain a training set for the joint behavior is not targeted. The problem is that all source trainings sets are independent random samples and thus the resulting training set exhibits a density (of operable power levels) that results from folding the source distributions.

Figure 3(a) shows a first example. Rather uniformly except for the gap between zero and minimum engine velocity and a slightly degrading likelihood of higher power levels distributed values for levels of power as in the case of an cogeneration plant with sufficient buffer capacity fold up to an multi-modal Irvin-Hall-distribution [37]. This distribution has some similarities to a sharp normal distribution and the more 


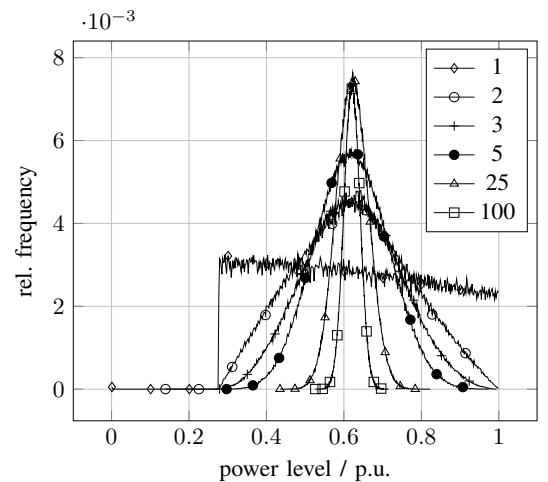

(a)

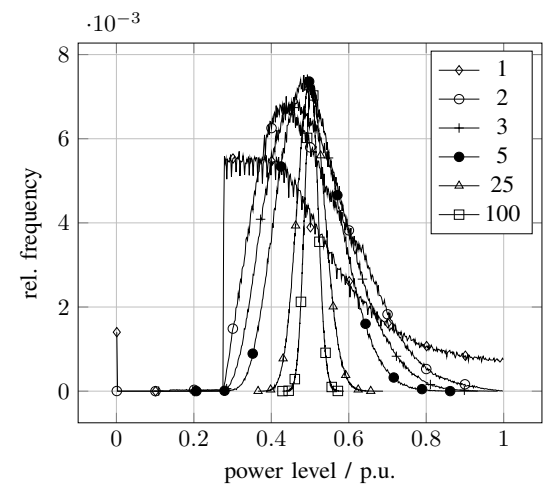

(b)

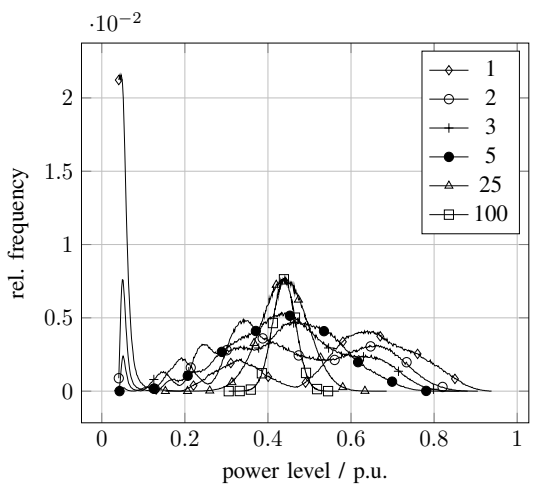

(c)

Fig. 3. Probability density of different numbers of folded distributions of operable power levels for co-generation plants for a very cold winters day.

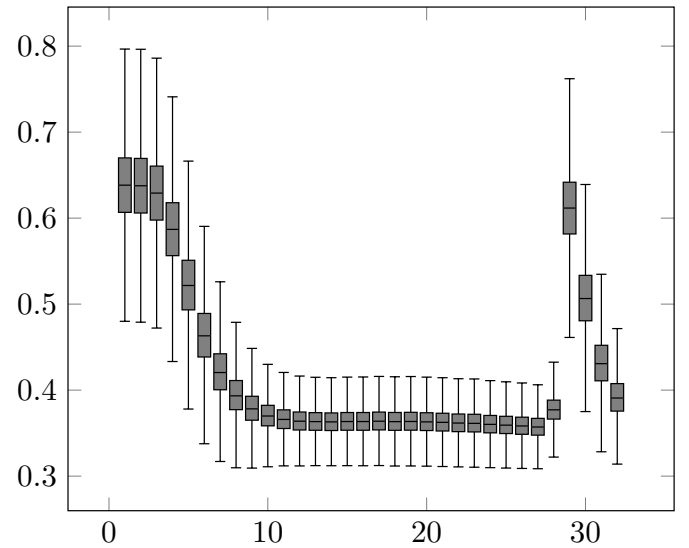

Fig. 4. Probability distribution of power levels at different time intervals of an ensemble of 10 micro CHP units. The training set exhibits a concentration in the inner part of the whole flexibility (grey boxes denoting $3 / 4$ of the samples) making it highly imbalanced.

samples (number of energy units in the ensemble) are folded the more leptokurtic the pdf gets.

Whereas Figure 3(a) considers the distribution of power levels at a certain point in time (7:00 a.m.), shows Figure 3(b) the situation averaged over all time periods of a sample winters day. Due to the integration of the warmer daylight periods, the likelihood of high power levels degrades. Nevertheless, in the case of ensembles of co-generation plants the distributions fold up to a similar aggregated distribution that generates training sets unsuitable for machine learning. If a model is based on an estimation of probability distributions, then it is highly susceptible to the spatial distribution of the samples in feasible space [38] because merely regions of high density are learned. Figure 3(c) shows as a third example the folded distributions in the case of a heat pump.

This folding leads to a sample with a very high density in the middle of the feasible region. At the outskirts the sample is extremely sparse. Thus, almost all instances from the outer parts are neglected as outliers from the support vector approach that generates the surrogate model and the decoder.

For this reason, a decoder trained from such a training sample reproduces only a very small, inner portion of the feasible region. In this way, most of the flexibility that an ensemble could bring in into virtual power plant control is neglected. This can also be seen in Figure 4. The rather small grey boxes represent the data (power levels for different time intervals) that actually should spread over the area denoted by the outer whiskers. Only the small inner part is going to be learned by a model.

\section{B. CMAES with decoder}

The covariance matrix adaption evolution strategy [39], [40] (CMA-ES) is a well known evolution strategy for solving multi modal black box problems.

CMA-ES improves its operations by harnessing lessons learned from previously successful evolution steps for future search directions. A new population of solution candidates is sampled from a multi variate normal distribution $\mathcal{N}(0, \boldsymbol{C})$ with covariance matrix $C$ which is adapted such that it that it maximizes the occurrence of improving steps according to previously seen distributions for good steps. Sampling offspring is weighted by a selection of solutions of the parent generation. In a way, the method learns a second order model of the objective function and exploits it for structure information and for reducing calls of objective evaluations. An a priori parametrization with structure knowledge of the problem by the user is not necessary as the method is capable of adapting unsupervised. A good introduction can for example be found in [41]. Especially for non-linear, non-convex black-box problems, the approach has demonstraded excellent performance [41]. CMAES is initially not designed for integrated constraint handling in constrained optimization. Nevertheless, some approaches for integrating constraint handling have been developed. In [42] a CMA-ES is introduced that learns constraint function models and rotates mutation distributions accordingly. In [43] an approximation of the directions of the local normal vectors of the constraint boundaries is built by accumulating steps that violate the respective constraints. Then, the variances of these directions are reduced for mutation.

CMA-ES is used for solving the internal optimization problem that arises when an agent has to decide on the best 
possible joint schedule to offer during the decision phase of the COHDA negotiation for virtual power plants. With our explanations we follow [22].

We consider an agent negotiation with a stage where each agent has to search the individual flexibility and thus the individual feasible region of operable schedules for the best option (according to given objectives). In case the agent has to control an ensemble with more the one local unit, a decoder that models the feasible region cannot be used as in the case of a single unit. For this reason, a local optimization problem has to be solved in order to decide on a schedule: find the closest aggregated schedule that the local ensemble can operate. This is basically the same problem as for predictive scheduling Eq. 1. As this smaller sub-problem happens to be a local one seen from the agent's perspective, there is no need to harness a distributed solving strategy. Additionally, the problem size is expected to stay rather small with a limited number of devices e. g. inside a household.

Because the operation of several decoders that model the different feasible regions of the local ensemble has to be involved, a heuristic that uses only a small number of objective evaluations is advantageous. CMA-ES is well known for this characteristic [41]. For handling the constraints, the readily available decoders can be used. Thus, the decoder technique also adapted to and employed to the CMA-ES part (cf. . [22]).

In each iteration $g$ of CMA-ES a multivariate distribution is sampled in order to generate a new offspring solution population in the vicinity of good parent solutions:

$$
\boldsymbol{x}_{k}^{(g+1)} \sim \boldsymbol{m}^{(g)}+\sigma^{(g)} \mathcal{N}\left(0, \boldsymbol{C}^{(g)}\right), k=1, \ldots, \lambda .
$$

$\boldsymbol{C}^{(g)} \in \mathbb{R}^{n \times n}$ constitutes the covariance matrix of the search distribution at generation (iteration) $g$ with overall standard deviation $\sigma^{(g)}$ which can also be interpreted in terms of an adaptive (multivariate) step size. The step size is adapted individually for each dimension to support and favor direction where fast improvement can be expected according to formerly seen results. The mean of the multivariate distribution is denoted by $\boldsymbol{m}^{(g)}, \lambda \geq 2$ denotes the population size.

The new mean $\boldsymbol{m}^{(g+1)}$ for generating the sample of the next generation in CMA-ES is calculated as weighted average

$$
\boldsymbol{m}^{(g+1)}=\sum_{i=1}^{\mu} w_{i} \boldsymbol{x}_{i: \lambda}^{(g+1)}, \sum w_{i}=1, w_{i}>0,
$$

of the best (in terms of objective function evaluation) individuals form the current sample $\boldsymbol{x}_{i}^{(g)}, \ldots, \boldsymbol{x}_{\lambda}^{(g)}$. In order to introduce the decoder into CMA-ES, ranking is now done with the help of the decoder mapping $\gamma$ :

$$
f\left(\gamma\left(\boldsymbol{x}_{1: \lambda}^{(g)}\right)\right), \ldots, f\left(\gamma\left(\boldsymbol{x}_{\lambda: \lambda}^{(g)}\right)\right), \lambda \geq \mu,
$$

to define $\boldsymbol{x}_{i: \lambda}^{(g)}$ as the $i$ th ranked best individual.

For the case of the ensemble scheduling example, a solution candidate $\boldsymbol{x}$ is the concatenation of individual schedules

$$
\begin{aligned}
\boldsymbol{x} & =\boldsymbol{p}_{1} \boldsymbol{p}_{2} \ldots \boldsymbol{p}_{m} \\
& =\left(p_{11}, p_{12}, \ldots, p_{1 d}, p_{21}, \ldots, p_{2 d}, \ldots, p_{m d}\right)
\end{aligned}
$$

with $\boldsymbol{p}_{1}, \ldots, \boldsymbol{p}_{m}$ denoting schedules for the respective units in the ensemble.

Finally, the covariance matrix is updated as usual, but also based on the decoder based ranking Eq. 6:

$$
\boldsymbol{C}_{\mu}^{(g+1)}=\sum_{i=1}^{\mu} w_{i}\left(\boldsymbol{x}_{i: \lambda}^{(g+1)}-m^{(g)}\right)\left(\boldsymbol{x}_{i: \lambda}^{(g+1)}-m^{(g)}\right)^{\top} .
$$

CMA-ES has a set of parameters that can be tweaked to some degree for a problem specific adaption. Nevertheless, default values that are applicable for a wide range of problems are usually available. For our experiments, we used the following default settings for the CMA-ES part. The (external) strategy parameters are $\lambda, \mu, w_{i=1 \ldots \mu}$, controlling selection and recombination; $c_{\sigma}$ and $d_{\sigma}$ for step size control and $c_{c}$ and $\mu_{\text {cov }}$ controlling the covariance matrix adaption. We have chosen to set these values after [41]:

$$
\begin{gathered}
\lambda=4+\lfloor 3 \ln n\rfloor, \quad \mu=\left[\frac{\lambda}{2}\right], \\
\boldsymbol{w}_{i}=\frac{\ln \left(\frac{\lambda}{2}+0.5\right)-\ln i}{\left.\sum_{\mu}^{j=1} \frac{\lambda}{2}+0.5\right)-\ln i}, i=1, \ldots, \mu \\
C_{c}=\frac{4}{n+4}, \mu_{\mathrm{cov}}=\mu_{e f f}, \\
C_{\mathrm{cov}}=\frac{1}{\mu_{\mathrm{cov}}} \frac{2}{(n+\sqrt{2})^{2}} \\
+\left(1-\frac{1}{\mu_{\mathrm{cov}}}\right) \min \left(1, \frac{2 \mu_{\mathrm{cov}}-1}{(n+2)^{2}+\mu_{\mathrm{cov}}}\right) .
\end{gathered}
$$

An in-depth discussion of these parameters is also given in [44]. These settings are specific to the dimension $N$ of the objective function. In our case is $N=d \cdot m$ related to the number of agents and the dimension of the assigned schedules in the test cases that are discussed in the following section.

\section{RESUlts}

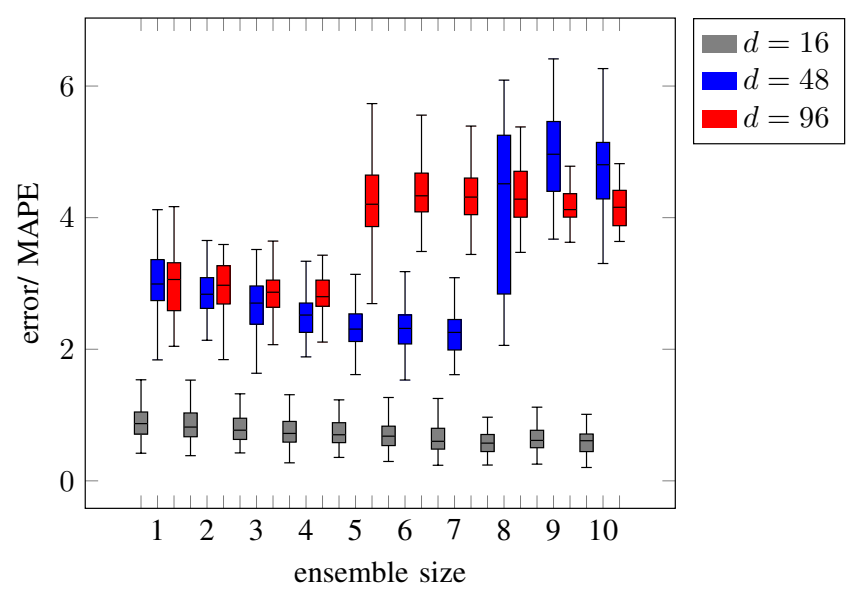

Fig. 5. Sensitivity of the approach to the group size (1 denotes no ensemble in the VPP) of an embedded ensemble in the VPP for different planning time horizons $d$. 


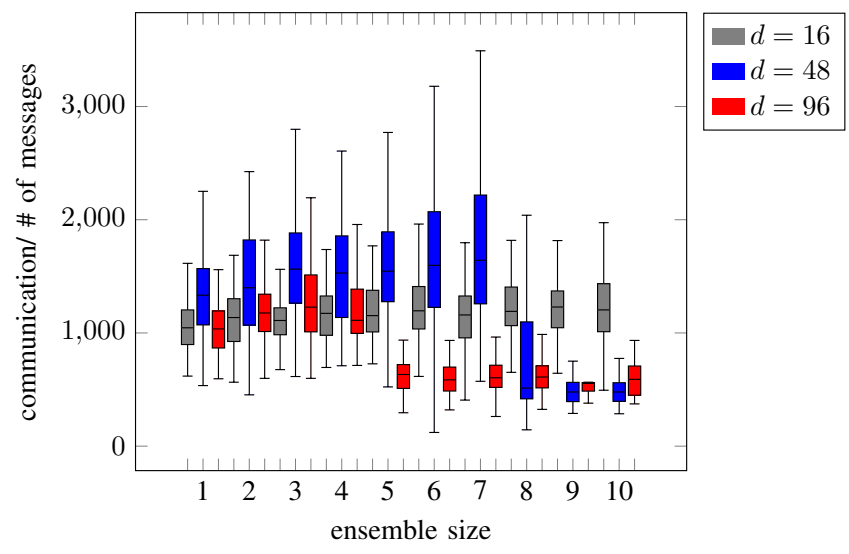

Fig. 6. Impact of the size of an embedded ensemble on the number of exchanges messages and thus on the number of local decisions for different planning horizons $d$.

TABLE I

SENSITIVITY OF THE APPROACH TO THE NUMBER OF GROUPS (SHARE OF THE WHOLE VPP IN PERCENT) AND IMPACT ON THE COMMUNICATION EFFORT (AND THUS ON LOCAL NUMBER OF DECISIONS) FOR A VPP WITH 10 PARTICIPANTS (SINGLE UNITS AND ENSEMBLES). SCHEDULING HAS BEEN SIMULATED WITH 96-DIMENSIONAL SCHEDULES FOR A PLANNING PERIOD OF A WHOLE DAY.

\begin{tabular}{c|cc}
\hline ensembles/ \% & $\delta_{M A P E}$ & \# messages \\
\hline \hline 0 & $2.531 \pm 1.254$ & $1055.50 \pm 244.15$ \\
2 & $2.672 \pm 0.626$ & $1271.16 \pm 312.46$ \\
4 & $2.502 \pm 0.289$ & $1531.94 \pm 350.78$ \\
6 & $2.352 \pm 0.231$ & $1613.08 \pm 389.72$ \\
8 & $2.204 \pm 0.210$ & $1629.28 \pm 422.94$ \\
10 & $2.102 \pm 0.231$ & $1581.00 \pm 413.47$ \\
\hline
\end{tabular}

Evaluation was again done by simulation with a setup comprising a set of simulated energy resources and a multi-agent system for control [22]. The agent system was implemented after [20]. Each agent is responsible for conducting local decisions and communication with other agents in charge of controlling a small local ensemble of jointly controlled energy resources. Each agent is equipped with the described CMA-ES approach for local decisions on operation.

As a model for distributed energy resources we used a model for co-generation plants that has already served in several studies and projects for evaluation [15], [30], [31], [45], [46]. This model comprises a micro CHP with $4.7 \mathrm{~kW}$ of rated electrical power $(12.6 \mathrm{~kW}$ thermal power) bundled with a thermal buffer store. Constraints restrict power band, buffer charging, gradients, min. on and off times, and satisfaction of thermal demand. Thermal demand is determined by simulating losses of a detached house (including hot water drawing) according to given weather profiles. For each agent the model is individually (randomly) configured with state of charge, weather condition, temperature range, allowed operation gradients, and similar. From these model instances, the respective training sets for building the decoders have been generated with the sampling approach from [36]. In addition, we used models for heat pumps and boilers for hot water provision [47]. A fourth model simulates the flexibilities of a cool storage.
TABLE II

SENSITIVITY OF THE APPROACH TO THE NUMBER OF GROUPS AND IMPACT ON THE COMMUNICATION EFFORT FOR A VPP WITH 50 PARTICIPANTS.

\begin{tabular}{c|cr}
\hline ensembles/\% & $\delta_{M A P E}$ & \# messages \\
\hline \hline 0 & $0.935 \pm 0.122$ & $87197.24 \pm 14149.94$ \\
20 & $0.900 \pm 0.354$ & $121473.82 \pm 49077.28$ \\
40 & $0.873 \pm 0.337$ & $130408.06 \pm 63458.05$ \\
60 & $0.770 \pm 0.350$ & $145282.74 \pm 86664.75$ \\
80 & $0.704 \pm 0.378$ & $156506.48 \pm 81141.94$ \\
100 & $0.795 \pm 0.500$ & $111301.94 \pm 78934.29$ \\
\hline
\end{tabular}

TABLE III

SENSITIVITY OF THE APPROACH TO THE NUMBER OF GROUPS AND IMPACT ON THE COMMUNICATION EFFORT FOR A VPP WITH 100 PARTICIPANTS.

\begin{tabular}{c|cc}
\hline ensembles/ \% & $\delta_{M A P E}$ & \# messages \\
\hline \hline 0 & $0.739 \pm 0.556$ & $404597.44 \pm 192869.14$ \\
20 & $0.776 \pm 0.425$ & $443387.92 \pm 419493.10$ \\
40 & $0.609 \pm 0.355$ & $488633.46 \pm 387336.30$ \\
60 & $0.591 \pm 0.347$ & $501521.85 \pm 573805.91$ \\
80 & $0.632 \pm 0.497$ & $493369.70 \pm 487284.04$ \\
100 & $0.987 \pm 0.660$ & $303466.90 \pm 412400.43$ \\
\hline
\end{tabular}

The applicability of the hybridized CMA-ES has already been demonstrated in [22]. The approach is able to achieve optimization results with a residual error less than 1 percent. Often well better results with an absolute error of about $30 \mathrm{~W}$ for scenarios with a rated power of $470 \mathrm{~kW}$ are achieved. Here, again we used the mean absolute percentage error (MAPE)

$$
\delta_{M A P E}=\delta(\boldsymbol{x}, \boldsymbol{\zeta})=\frac{100}{d} \sum_{i=1}^{d}\left|\frac{\zeta_{i}-x_{i}}{\zeta_{i}}\right|,
$$

in order to be able to compare different scenarios with different number of energy units and different rated power.

We simulated the effect of integrating ensembles instead of single energy units into a VPP on the residual error and on the number of exchanged messages between the agents. As the agent system under research is a gossiping type of agent system [48], each message triggers a local decision that translates into a decoder call in the single unit case but into solving a optimization problem with CMA-ES in the ensemble case. Thus, the number of messages is an important indicator for performance scaling with number of integrated ensembles.

Figure 5 shows a first result. The experiment scrutinized a VPP with 10 participants. One participant is an ensemble. The size of the ensemble has been increased from 1 to 10 (an ensemble of size 1 translates again to a single unit) to evaluate means residual error and number of exchanged messages. The experiment has been conducted for differently large planning horizons. For shorter planning horizons the size of the ensemble has almost no impact. Actually, the residual error decreases a little (due to growing flexibility in the VPP). For longer planning horizons the same effect can be observed up to a size where the error escalates to a higher level. At the same time, the number of exchanged messages decreases (cf. Fig. 6). Obviously, the CMA-ES approach starts suffering from some premature convergence problems when the local problem size (ensemble size time schedule dimension) exceeds a certain size; at least when the standard parametrization is 
used. Premature convergence at the second level optimization inside an ensemble leads to similar results in successive optimization attempts with similar schedule configurations in the whole VPP. As no better solution is found, the agent sends no message and the first level optimization at agent level ceases earlier with a sub-optimal solution. Hence, integrating methods to prevent premature convergence in the CMA-ES part could largely improve the whole VPP optimization in case of larger ensemble sizes.

Another experiment scrutinizes the number of ensembles in a VPP. Tables I to III show the result. Now, the share of ensembles (with a fixed size of $3 \mathrm{CHP}$ ) in a VPP is varying from 0 to 100 percent and the effect is scrutinized. The result quality increases in most of the cases due to a growing flexibility with a growing number of ensembles. For smaller VPP sizes the communication effort grows with the number of ensembles, for larger VPPs the number of sent messages stays on the same level compared with the case of 100 percent single units.

With these results, one can conclude that the introduction of ensembles does not deteriorate the performance of the agentbased predictive scheduling. Performance shortcomings for larger ensembles seem to be due to premature convergence and should be overcome with future integration of e.g. a better adapted step size control.

\section{CONCLUSION}

Using machine learning approaches for flexibility modeling and automatically deriving decoders from these models for efficient and domain knowledge independent implementation of (distributed) optimization methods has proven a useful tool in managing the future smart grid. So far, these models can only be applied to single energy units, because distributions of power levels in the training sets of single units fold up when aggregating them to ensemble training sets. Thus, the training set renders useless for appropriately learning a model for the joint flexibility of a group of energy units.

[22] presented a hybrid approach to overcome the problem of folded densities when training decoders for ensembles of energy resources in predictive scheduling. To achieve this, we embedded a CMA-ES solver in the decision routine of an established agent-based solution.

With this approach also households, hotels, small businesses, schools or similar with an ensemble of co-generation, heat pump, solar power, and controllable consumers can take part in agent-based decentralized predictive scheduling for providing energy services in future smart grid architectures without a need for an (expensive) individual link of each single device in the ensemble. By using a hybrid approach of evolution strategy and support vector based decoder, such ensemble based participants in virtual power plants can easily be represented by a single agent. Moreover, agents with our decision method still implement the same interface as single unit agents and can thus be easily integrated with the standard COHDA protocol. Applicability had already been demonstrated in [22].
Our new simulations showed that CMA-ES is well suitable for being hybridized with a decoder in order to build a system that may operate with arbitrary energy units regardless of individual constraints that restrict feasible operation. CMA-ES performs satisfactorily on reasonable large ensembles. Additional simulations showed that size and number of ensembles within a VPP scale well up to reasonable sizes. Communication does not suffer from an increase in number of exchanged messages. Based on these results the inclusion of secondary, local optimization objectives like cost or preferences are a consequentially next step in future work.

\section{REFERENCES}

[1] European Parliament \& Council, "Directive 2009/28/ec of 23 april 2009 on the promotion of the use of energy from renewable sources and amending and subsequently repealing directives 2001/77/ec and 2003/30/ec."

[2] O. Abarrategui, J. Marti, and A. Gonzalez, "Constructing the active european power grid," in Proceedings of WCPEE09, Cairo, 2009.

[3] A. Nieße, S. Lehnhoff, M. Trschel, M. Uslar, C. Wissing, H. J. Appelrath, and M. Sonnenschein, "Market-based self-organized provision of active power and ancillary services: An agent-based approach for smart distribution grids," in Complexity in Engineering (COMPENG), 2012, June 2012, pp. 1-5.

[4] K. Vinay Kumar and R. Balakrishna, "Smart grid: Advanced metering infrastructure (ami) \& distribution management systems (dms)," International Journal of Computer Science and Engineering, vol. 3, no. 11, 2015.

[5] I. Colak, G. Fulli, S. Sagiroglu, M. Yesilbudak, and C.-F. Covrig, "Smart grid projects in europe: Current status, maturity and future scenarios," Applied Energy, vol. 152, pp. 58 - 70, 2015.

[6] S. Awerbuch and A. M. Preston, Eds., The Virtual Utility: Accounting, Technology \& Competitive Aspects of the Emerging Industry, ser. Topics in Regulatory Economics and Policy. Kluwer Academic Publishers, 1997, vol. 26.

[7] M. Sonnenschein, O. Lünsdorf, J. Bremer, and M. Tröschel, "Decentralized control of units in smart grids for the support of renewable energy supply," Environmental Impact Assessment Review, no. 0, pp. -, 2014, in press.

[8] R. Kamphuis, C. Warmer, M. Hommelberg, and K. Kok, "Massive coordination of dispersed generation using powermatcher based software agents," in 19th International Conference on Electricity Distribution, 05 2007.

[9] K. Kok, Z. Derzsi, J. Gordijn, M. Hommelberg, C. Warmer, R. Kamphuis, and H. Akkermans, "Agent-based electricity balancing with distributed energy resources, a multiperspective case study," Hawaii International Conference on System Sciences, vol. 0, p. 173, 2008.

[10] A. Kamper and A. Esser, "Strategies for decentralised balancing power," in Biologically-inspired Optimisation Methods: Parallel Algorithms, Systems and Applications, ser. Studies in Computational Intelligence, M. R. A. Lewis, S. Mostaghim, Ed. Berlin, Heidelberg: Springer, Juni 2009, no. 210, pp. 261-289.

[11] R.-C. Mihailescu, M. Vasirani, and S. Ossowski, "Dynamic coalition adaptation for efficient agent-based virtual power plants," in Proceedings of the 9th German conference on Multiagent system technologies, ser. MATES'11. Berlin, Heidelberg: Springer-Verlag, 2011, pp. 101-112.

[12] S. D. Ramchurn, P. Vytelingum, A. Rogers, and N. R. Jennings, "Agentbased control for decentralised demand side management in the smart grid," in AAMAS, L. Sonenberg, P. Stone, K. Tumer, and P. Yolum, Eds. IFAAMAS, 2011, pp. 5-12.

[13] J. Bremer, B. Rapp, and M. Sonnenschein, "Support vector based encoding of distributed energy resources' feasible load spaces," in IEEE PES Conference on Innovative Smart Grid Technologies Europe, Chalmers Lindholmen, Gothenburg, Sweden, 2010.

[14] J. Bremer and M. Sonnenschein, "A distributed greedy algorithm for constraint-based scheduling of energy resources," in Federated Conference on Computer Science and Information Systems - FedCSIS 2012 Wroclaw, Poland, 9-12 September 2012, Proceedings, M. Ganzha, L. A. Maciaszek, and M. Paprzycki, Eds., 2012, pp. 1285-1292. 
[15] — "Constraint-handling for optimization with support vector surrogate models - a novel decoder approach," in ICAART 2013 - Proceedings of the 5th International Conference on Agents and Artificial Intelligence, J. Filipe and A. Fred, Eds., vol. 2. Barcelona, Spain: SciTePress, 2013, pp. 91-105.

[16] A. Nieße and M. Sonnenschein, "A fully distributed continuous planning approach for decentralized energy units," in Multiagent System Technologies, ser. Lecture Notes in Computer Science. Springer Berlin Heidelberg, 2015.

[17] A. Nieße, S. Beer, J. Bremer, C. Hinrichs, O. Lünsdorf, and M. Sonnenschein, "Conjoint dynamic aggrgation and scheduling for dynamic virtual power plants," in Federated Conference on Computer Science and Information Systems - FedCSIS 2014, Warsaw, Poland, M. Ganzha, L. A. Maciaszek, and M. Paprzycki, Eds., 92014.

[18] J. Bremer and M. Sonnenschein, "Parallel tempering for constrained many criteria optimization in dynamic virtual power plants," in Computational Intelligence Applications in Smart Grid (CIASG), 2014 IEEE Symposium on, Dec 2014, pp. 1-8.

[19] A. Schiendorfer, J.-P. Steghöfer, and W. Reif, "Synthesised constraint models for distributed energy management," in Proceedings of the 2014 Federated Conference on Computer Science and Information Systems, Warsaw, Poland, September 7-10, 2014, M. Ganzha, L. A. Maciaszek, and M. Paprzycki, Eds., 2014, pp. 1529-1538.

[20] C. Hinrichs, "Selbstorganisierte Einsatzplanung dezentraler Akteure im Smart Grid," Ph.D. dissertation, Carl von Ossietzky Universitt Oldenburg, 2014.

[21] J. Bremer and S. Lehnhoff, "Decentralized coalition formation in agentbased smart grid applications," in Highlights of Practical Applications of Scalable Multi-Agent Systems. The PAAMS Collection, ser. Communications in Computer and Information Science, vol. 616. Springer, 2016, pp. 343-355.

[22] — Hybrid Multi-ensemble Scheduling. Cham: Springer International Publishing, 2017, pp. 342-358.

[23] S. McArthur, E. Davidson, V. Catterson, A. Dimeas, N. Hatziargyriou, F. Ponci, and T. Funabashi, "Multi-agent systems for power engineering applications - Part I: Concepts, approaches, and technical challenges," IEEE Transactions on Power Systems, vol. 22, no. 4, pp. 1743-1752, 2007.

[24] M. Sonnenschein, C. Hinrichs, A. Nieße, and U. Vogel, "Supporting renewable power supply through distributed coordination of energy resources," in ICT Innovations for Sustainability, ser. Advances in Intelligent Systems and Computing, L. M. Hilty and B. Aebischer, Eds Springer International Publishing, 2015, vol. 310, pp. 387-404.

[25] F. Gieseke and O. Kramer, "Towards non-linear constraint estimation for expensive optimization," in Applications of Evolutionary Computation ser. Lecture Notes in Computer Science, A. Esparcia-Alcázar, Ed. Springer Berlin Heidelberg, 2013, vol. 7835, pp. 459-468.

[26] J. Bremer and M. Sonnenschein, "Model-based integration of constrained search spaces into distributed planning of active power provision." Comput. Sci. Inf. Syst., vol. 10, no. 4, pp. 1823-1854, 2013.

[27] C. A. Coello Coello, "Theoretical and numerical constraint-handling techniques used with evolutionary algorithms: a survey of the state of the art," Computer Methods in Applied Mechanics and Engineering, vol. 191, no. 11-12, pp. 1245-1287, Jan. 2002.

[28] C. Hinrichs, M. Sonnenschein, and S. Lehnhoff, "Evaluation of a SelfOrganizing Heuristic for Interdependent Distributed Search Spaces," in International Conference on Agents and Artificial Intelligence (ICAART 2013), J. Filipe and A. L. N. Fred, Eds., vol. Volume 1 - Agents. SciTePress, 2013, pp. 25-34.

[29] C. Hinrichs, S. Lehnhoff, and M. Sonnenschein, "A Decentralized Heuristic for Multiple-Choice Combinatorial Optimization Problems," in Operations Research Proceedings 2012. Springer, 2014, pp. 297 302 .
[30] C. Hinrichs, J. Bremer, and M. Sonnenschein, "Distributed Hybrid Constraint Handling in Large Scale Virtual Power Plants," in IEEE PES Conference on Innovative Smart Grid Technologies Europe (ISGT Europe 2013). IEEE Power \& Energy Society, 2013.

[31] A. Nieße and M. Sonnenschein, "A fully distributed continuous planning approach for decentralized energy units," in Informatik 2015. GI-Edition - Lecture Notes in Informatics (LNI), D. W. Cunningham, P. Hofstedt, K. Meer, and I. Schmitt, Eds., vol. 246. Bonner Köllen Verlag, 2015 , pp. 151-165.

[32] R. Poli, J. Kennedy, and T. Blackwell, "Particle swarm optimization," Swarm Intelligence, vol. 1, no. 1, pp. 33-57, 2007.

[33] D. Karaboga and B. Basturk, "A powerful and efficient algorithm for numerical function optimization: artificial bee colony (ABC) algorithm," Journal of Global Optimization, vol. 39, no. 3, pp. 459-471, Nov. 2007.

[34] T. Lust and J. Teghem, "The multiobjective multidimensional knapsack problem: a survey and a new approach," CoRR, vol. abs/1007.4063, 2010.

[35] D. Watts and S. Strogatz, "Collective dynamics of 'small-world' networks," Nature, vol. 393, no. 6684, pp. 440-442, 1998.

[36] J. Bremer and M. Sonnenschein, "Sampling the search space of energy resources for self-organized, agent-based planning of active power provision," in 27th International Conference on Environmental Informatics for Environmental Protection, EnviroInfo 2013, B. Page, A. G. Fleischer, J. Göbel, and V. Wohlgemuth, Eds. Shaker, 2013, pp. 214-222.

[37] P. Hall, "The distribution of means for samples of size $n$ drawn from a population in which the variate takes values between 0 and 1, all such values being equally probable," Biometrika, vol. 19 , no. $3 / 4$, pp. pp. 240-245, 1927.

[38] D. M. J. Tax and R. P. W. Duin, "Support vector data description," Mach. Learn., vol. 54, no. 1, pp. 45-66, 2004.

[39] A. Ostermeier, A. Gawelczyk, and N. Hansen, "A derandomized approach to self-adaptation of evolution strategies," Evolutionary Computation, vol. 2, no. 4, pp. 369-380, 1994.

[40] N. Hansen, "The CMA evolution strategy: a comparing review," in Towards a new evolutionary computation. Advances on estimation of distribution algorithms, J. Lozano, P. Larranaga, I. Inza, and E. Bengoetxea, Eds. Springer, 2006, pp. 75-102.

[41] — "The CMA Evolution Strategy: A Tutorial," Tech. Rep., 2011.

[42] O. Kramer, A. Barthelmes, and G. Rudolph, "Surrogate constraint functions for cma evolution strategies," in Proceedings of the $32 \mathrm{Nd}$ Annual German Conference on Advances in Artificial Intelligence, ser. KI'09. Berlin, Heidelberg: Springer-Verlag, 2009, pp. 169-176.

[43] D. V. Arnold and N. Hansen, "A (1+1)-cma-es for constrained optimisation," in Proceedings of the 14th Annual Conference on Genetic and Evolutionary Computation, ser. GECCO '12. New York, NY, USA: ACM, 2012, pp. 297-304.

[44] N. Hansen and A. Ostermeier, "Completely derandomized selfadaptation in evolution strategies," Evol. Comput., vol. 9, no. 2, pp. 159-195, Jun. 2001.

[45] J. Bremer, B. Rapp, and M. Sonnenschein, "Encoding distributed search spaces for virtual power plants," in IEEE Symposium Series on Computational Intelligence 2011 (SSCI 2011), Paris, France, 42011.

[46] J. Neugebauer, O. Kramer, and M. Sonnenschein, "Classification cascades of overlapping feature ensembles for energy time series data," in Proceedings of the 3rd International Workshop on Data Analytics for Renewable Energy Integration (DARE'15). Springer, 2015.

[47] M. Sonnenschein, H.-J. Appelrath, W.-R. Canders, M. Henke, M. Uslar, S. Beer, J. Bremer, O. Lünsdorf, A. Nieße, J.-H. Psola et al., "Decentralized provision of active power," in Smart Nord - Final Report. Hartmann GmbH, Hannover, 2015.

[48] S. Boyd, A. Ghosh, B. Prabhakar, and D. Shah, "Gossip algorithms: Design, analysis and applications," in Proceedings IEEE 24th Annual Joint Conference of the IEEE Computer and Communications Societies., vol. 3. IEEE, 2005, pp. 1653-1664. 